\title{
N88: The physical properties of a compact star-forming region and its neighbourhood in the SMC ${ }^{\star}$
}

\section{II. $\mathrm{H}$ and $\mathrm{H}_{2}$ near infrared emission}

\author{
G. Testor $^{1, * *}$, J. L. Lemaire ${ }^{2, \star \star}$, D. Field ${ }^{3}$, and G. Callejo ${ }^{2}$ \\ 1 LUTH, UMR 8102 du CNRS, Observatoire de Paris, 92195 Meudon, France \\ e-mail: gerard.testor@obspm.fr \\ 2 LERMA, UMR 8112 du CNRS, Observatoire de Paris, 92195 Meudon, France \\ and Université de Cergy-Pontoise, 95031 Cergy Cedex, France \\ e-mail: jean-louis.lemaire@obspm.fr \\ 3 Department of Physics and Astronomy, Århus University, 8000 Århus C, Denmark \\ e-mail: dfield@phys.au.dk
}

Received 6 April 2004 / Accepted 14 December 2004

\begin{abstract}
This paper, the second in a series of papers on the conspicuous compact HII region N88A in the Small Magellanic Cloud, emphasizes the properties of the $\mathrm{H}_{2}$ emission as well as of the surrounding stellar content of the entire Henize region N88. Using VJK photometry and stellar evolutionary models our analysis of N88 reveals a miscellaneous population of evolved stars of low and intermediate masses $\left(1.2-8 M_{\odot}\right)$ in an age range of $40 \mathrm{Myr}$ to $6 \mathrm{Gyr}$. The majority of these stars are found in the cluster HW 82 which also contains several main sequence stars. $A_{\mathrm{V}}$ in N88A is revisited using near-IR $\mathrm{P} \delta, \mathrm{P} \gamma$ and $\mathrm{P} \beta$ Paschen emission lines. Spectroscopic measurements of the $\mathrm{H}_{2}$ emission in $Z, J, H$ and $K$ bands allow, in conjunction with measurements in the visible, a full spectral coverage from $0.37 \mu \mathrm{m}$ to $2.45 \mu \mathrm{m}$. Long slit spectroscopy through N88 and the nebulosity to the east clearly distinguishes the ionization zone in $\mathrm{N} 88$ characterized by $\mathrm{H} \mathrm{I}$ and He I emission with pure $\mathrm{H}_{2}$ emission clearly delineated in the cloud nearby. The principal excitation mechanism for $\mathrm{H}_{2}$ is through fluorescent excitation in a photodissociation region associated with N88A.
\end{abstract}

Key words. Magellanic Clouds - stars: formation - circumstellar matter - ISM: molecules - infrared: stars - stars: evolution

\section{Introduction}

We present near infrared observations of the compact star forming region N88, using the European Southern Observatory Very Large Telescope (ESO-VLT). This paper complements an earlier publication related to the optical study of N88A and its immediate environment as well as the associated $\mathrm{H}_{2}$ emission (Testor et al. 2003, hereafter Paper I). The extended region N88 (Henize 1956) (designated as LHA $115-\mathrm{N} 88$ in Simbad) is a complex of scale $2.8^{\prime} \times 1.5^{\prime}$ in the Small Magellanic Cloud (SMC) where the conspicuous region N88A is found. It is the brightest compact HII region $(\sim 2$ '. 4$)$ of the six similar ones known in the SMC (Testor \& Pakull 1985; Heydari-Malayeri et al. 1988; Testor 2001; Stanghellini et al. 2003). Such compact HII regions are generally characterized by high extinction and are ionized by a tight cluster or a single star. They belong to a rare class of compact objects called High- Excitation Blobs or HEBs (Heydari \& Testor 1982). These objects and their

^ Based on observations obtained at the European Southern Observatories, El Paranal, Chile.

$\star \star$ Visiting astronomer at VLT Paranal. environment offer insights into the early stages of massive star formation in a low metallicity environment.

N88 is formed by the clusters HW 81, HW 82 (Hodge \& Wright 1974) and the HEB N88A. HW 81, with a size of about $60^{\prime \prime} \times 40^{\prime \prime}$, is composed of at least seven single or complex OB stars of type O3-4 to B0 III (Wilcots 1994, Paper I). The HW 81 region appears exposed to UV from the dusty core of N88A, whose spectral type, equivalent to an O3-4 V star, was derived by a photoionization model. To the north of N88A our $V-K$ photometry of the HW 81 cluster shows an evolved star of type G-K III. Paper I also gives the integrated extinction $A_{\mathrm{V}}=1.38$ derived from the $\mathrm{H} \alpha / \mathrm{H} \beta$ ratio as well as an accurate morphology of the $\mathrm{H}_{2}$ emission. This emission obtained from CCD imagery extends up to $3^{\prime \prime}-6$ " from the central N88A core with a good $S / N$ ratio and with fainter traces up to $20^{\prime \prime}-24^{\prime \prime}$. The cluster HW 82 is a mix of old and young stars not associated with ionized gas (Heydari-Malayeri et al. 1999, hereafter HM99).

Israel \& Koorneef (1988) have detected the presence of molecular hydrogen in $\mathrm{N} 88$ through $\mathrm{H}_{2}$ emission. The authors 
Table 1. Log of the VLT ISAAC long-slit spectroscopic observations.

\begin{tabular}{|c|c|c|c|c|c|c|c|c|}
\hline Direction & Date & $\begin{array}{l}\text { Slit } \\
\prime \prime\end{array}$ & Spectra & $\begin{array}{l}\operatorname{Expo}^{f} \\
\text { (s) }\end{array}$ & Mode & $\lambda / \delta \lambda$ & $\begin{array}{c}\text { Seeing } \\
\prime \prime\end{array}$ & Centering $^{c}$ \\
\hline \multirow[t]{4}{*}{$\mathbf{a}$} & $12 / 7 / 2002$ & 2 & $K(1.82-2.56 \mu \mathrm{m})$ & $2 \times 5 \times 100$ & SW-LR & 250 & 1.3 & $\left(\mathrm{PA}=99^{\circ}\right)$ \\
\hline & & & $H(1.4-1.82 \mu \mathrm{m})$ & & & 280 & & \\
\hline & & & $J(1.1-1.4 \mu \mathrm{m})$ & & & 310 & & \\
\hline & & & $Z(0.98-1.1 \mu \mathrm{m})$ & $2 \times 3 \times 100$ & & 350 & & \\
\hline \multirow[t]{2}{*}{$\mathbf{b}^{n}$} & $13 / 7 / 2002$ & 0.6 & $K(1.82-2.56 \mu \mathrm{m})$ & $2 \times 5 \times 60$ & SW-MR & 650 & 0.8 & $\left(\mathrm{PA}=121^{\circ}\right)$ \\
\hline & & & $H(1.4-1.82 \mu \mathrm{m})$ & $2 \times 3 \times 60$ & & 720 & & \\
\hline
\end{tabular}

${ }^{c}$ The slit crosses the center of N88A. In direction b two stars labelled \#24 and \#25 (Fig. 1) are visible at the bottom of the slit (Fig. 2).

${ }^{f}$ The factor 2 in the exposure time corresponds to the nodding and the second factor to the exposure number.

${ }^{n}$ These two spectra are incomplete due to instrumental failure.

propose that the observed $\mathrm{H}_{2}$ is either shock-excited on a small scale of 0.46 by stars embedded in the molecular cloud or radiatively excited on a large scale $\left(3^{\prime \prime}-6^{\prime \prime}\right)$. However their low spectral $(R=50)$ and spatial $\left(7 . .5-10^{\prime \prime}\right.$ aperture $)$ resolutions do not allow discrimination of these different processes. They describe N88A as a strong near infrared source dominated by nebular emission containing a strong hot dust component (Israel \& Koorneef 1991). Using SEST, Israel et al. (2003) have detected a large $\mathrm{CO}$ molecular cloud of $1.5^{\prime} \times 1.5^{\prime}$ in the region, reporting spectra and maps of the ${ }^{12} \mathrm{CO}$ lines $J=1-0$ and $J=2-1$.

In the present paper we extend the study of the stellar content to the whole field of N88 using mainly $V-K$ photometry. We revisit the integrated extinction of N88A using the $\mathrm{P} \delta, \mathrm{P} \gamma$ and $\mathrm{P} \beta$ Paschen emission lines. In addition, we study the $\mathrm{H}_{2}$ emission associated with N88A based on ZJHK spectroscopy along two differently oriented long slits and show that the $\mathrm{H}_{2}$ emission arises from a fluorescence mechanism in low density gas $\left(10^{3} \mathrm{~cm}^{-3}\right.$ or less).

The paper is organized as follows. In Sect. 2 we give a description of photometry and spectroscopic observations and details of data reduction. Section 3 gives the results on stellar content, extinction and the excitation mechanisms of $\mathrm{H}_{2}$ with a comparison with $\mathrm{CO}$ observations. Our conclusions are presented in Sect. 4.

\section{Observations and data reduction}

Observations of N88 were performed with the VLT UT1 during the night of 12th and 13th July 2002, for about one hour at the end of each night (Table 1). The seeing during these nights was variable, yielding images with a measured full width at half maximum $(F W H M)$ in the infrared of 0.8 to 1. .'3. The conditions were photometric. We used the Infrared Spectrometer and Array Camera (ISAAC).

\subsection{Photometry}

We extend the photometry of N88 to a larger field of view $\left(2.5^{\prime} \times 2.5^{\prime}\right)$ using the $K$ and cont 5300 images listed in Table 2 of Paper I. The field corresponds to the dimensions of the CCD frame (Fig. 1) and contains approximately the whole area of N88 (Davies et al. 1976). The method of deriving the

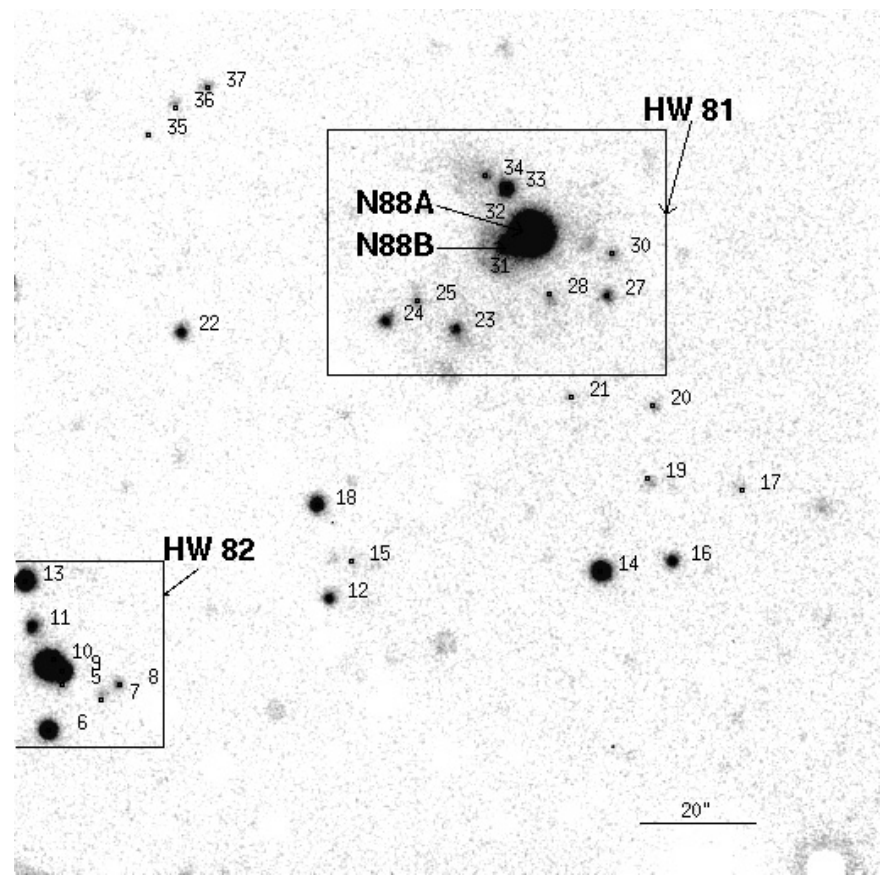

Fig. 1. Image of the compact star forming region $\mathrm{N} 88$ in the $K s$ band (background sky subtracted) corresponding to a field of $150^{\prime \prime} \times 150^{\prime \prime}$ $(48 \times 48 \mathrm{pc})$, showing the clusters HW 81 (to the north) and HW 82 (to the east) with \#11, which may be a cluster, see Sect. 3.1.2. The stars \#32 and \#31 correspond to the components N88A and N88B respectively.

instrumental magnitudes of the stars labelled in Fig. 1 and their photometric calibration is the same as described in Paper I. The results of our extended photometry in cont ${ }_{5300}$ and $K$ bands are listed in Table 2 (Col. 4: $\left(v_{\mathrm{c}}\right)^{p}$ and Col. 13: $\mathrm{K}^{p}$ ), which also gives the coordinates (J2000) (Cols. 2 and 3), and our $K$ s-image identification (Col. 1) from Fig. 1. In Table 2 the other columns show different photometries found in the literature. Columns 5 and 6 correspond to the $V$ and $B-V$ photometry of Massey (2002). Column 8 lists the high spatial resolution photometry in the Strömgren y (F547) band of HM99 (with labelling of HM99 in Col. 7) pointing out the complexity of the stars, particularly star \#11. Columns 9-12 show the $I, J, H$ and $K$ photometry extracted from DENIS (Cioni et al. 2000) and 2MASS 
Table 2. Optical and near NIR band photometry in the N88 region (field of $\left.2.5^{\prime} \times 2.5^{\prime}\right)$.

\begin{tabular}{|c|c|c|c|c|c|c|c|c|c|c|c|c|c|c|}
\hline $\mathrm{id}^{p}$ & $\begin{array}{c}\alpha \\
(2000)\end{array}$ & $\begin{array}{l}\left(v_{c}\right)^{p} \\
\mathrm{mag}\end{array}$ & $\begin{array}{l}V^{m} \\
\text { mag }\end{array}$ & $B-V^{m}$ & $\mathrm{id}^{h}$ & $\begin{array}{l}y^{h} \\
\text { mag }\end{array}$ & $\begin{array}{l}I^{d} \\
\mathrm{mag}\end{array}$ & $\begin{array}{l}J^{2 m} \\
\text { mag }\end{array}$ & $\begin{array}{l}H^{2 m} \\
\mathrm{mag}\end{array}$ & $\begin{array}{l}K^{2 m} \\
\mathrm{mag}\end{array}$ & $\begin{array}{l}K^{p} \\
\mathrm{mag}\end{array}$ & $M_{V}$ & $v_{\mathrm{c}}-K^{p}$ & $J^{2 m}-K^{p}$ \\
\hline 4 & $12429.5-731017.9$ & 16.80 & & & 77 & 18.05 & & & & & & -2.29 & & \\
\hline 5 & $12427.3-731024.2$ & 16.30 & & & 5 & 17.49 & & & & & & -2.79 & & \\
\hline 6 & $12427.3-731030.9$ & 17.28 & & & 76 & 18.18 & 15.96 & 14.85 & 14.19 & 14.07 & 13.95 & -1.81 & 3.30 & 0.90 \\
\hline 7 & $12424.7-731025.2$ & 15.43 & 14.94 & 0.45 & 4 & 16.78 & & & & & 16.43 & -3.66 & -1.0 & \\
\hline 8 & $12424.5-731023.1$ & & 15.53 & -0.56 & & & & 16.34 & 15.38 & 15.21 & 15.90 & & & 0.44 \\
\hline 9 & $12426.9-731020.6$ & 16.90 & & & 5 & 17.49 & & & & & 13.92 & -2.19 & 2.98 & \\
\hline 10 & $12427.3-731019.6$ & 14.89 & & & $\mathrm{c}^{1}$ & 15.53 & 13.85 & 13.04 & 12.40 & 12.29 & 12.27 & -4.20 & 2.62 & 0.77 \\
\hline 11 & $12428.0-731013.0$ & 13.71 & 13.71 & -0.02 & $c^{2}$ & 14.84 & 14.51 & 14.84 & 14.98 & 15.06 & 15.16 & -5.38 & -1.46 & -0.32 \\
\hline 12 & $12416.3-731008.1$ & & & & 17 & 17.48 & 16.20 & 15.76 & 15.32 & 15.02 & 15.12 & & & 0.64 \\
\hline 13 & $12428.3-731004.9$ & 14.66 & & & 19 & 15.62 & 14.40 & 13.96 & 13.58 & 13.67 & 13.22 & -4.43 & 1.44 & 0.74 \\
\hline 14 & $12405.2-731003.4$ & 16.96 & 17.04 & 1.06 & 74 & 17.84 & 15.81 & 14.76 & 13.93 & 13.80 & 13.72 & -2.13 & 3.24 & 1.04 \\
\hline 15 & $12415.8-731001.9$ & 15.86 & 15.18 & -0.11 & 22 & 17.16 & & & & & 16.60 & -3.22 & -0.74 & \\
\hline 16 & $12402.4-731001.5$ & 17.43 & & & 75 & 18.46 & & 15.79 & 15.48 & 15.01 & 15.11 & -1.66 & 2.32 & 0.68 \\
\hline 17 & $12359.4-730948.2$ & 15.43 & 16.27 & -0.13 & 26 & 16.90 & & & & & 16.81 & -3.66 & 1.38 & \\
\hline 18 & $12417.0-730953.0$ & & & & & & 16.67 & 15.43 & 14.88 & 14.86 & 14.56 & & & 0.87 \\
\hline 19 & $12402.9-730948.3$ & & 16.94 & -0.28 & & & & & & & 16.55 & -2.15 & 0.35 & \\
\hline 20 & $12402.9-730934.5$ & 15.57 & 16.24 & -0.10 & 30 & 16.97 & & 16.49 & 16.72 & 15.70 & 16.36 & -3.52 & -0.79 & 0.13 \\
\hline 21 & $12406.5-730933.0$ & 15.83 & & & 32 & 17.01 & & & & & 16.70 & -3.26 & -0.87 & \\
\hline 22 & $12422.0-730921.4$ & & & & & & & 16.02 & 15.45 & 15.45 & 15.26 & & & 0.76 \\
\hline 23 & $12410.9-730919.4$ & 14.82 & & & $c^{3}$ & 15.94 & 15.41 & 15.46 & 15.67 & 15.49 & 15.55 & -4.27 & -0.73 & -0.09 \\
\hline 24 & $12414.2-730920.5$ & 14.02 & 14.21 & -1.00 & 39 & 15.28 & 14.89 & 15.22 & 15.25 & 15.25 & 15.30 & -5.07 & -1.28 & -0.08 \\
\hline 25 & $12412.5-730915.9$ & 15.10 & & & 78 & 16.46 & 15.76 & 16.42 & 15.82 & 15.76 & 16.19 & -3.99 & -1.09 & 0.23 \\
\hline 27 & $12404.5-730914.1$ & 14.70 & 14.70 & -0.12 & $c^{4}$ & 15.68 & & 15.67 & 15.60 & 15.63 & 15.72 & -4.39 & -1.02 & -0.05 \\
\hline 28 & $12407.3-730915.0$ & 15.52 & & & $c^{5}$ & 16.41 & 15.76 & 16.54 & 15.20 & 15.14 & 16.43 & -3.57 & -0.91 & 0.11 \\
\hline 30 & $12404.4-730908.1$ & 15.14 & 14.67 & -0.18 & 73 & & & 16.13 & 15.93 & 15.68 & 16.25 & -3.95 & -1.11 & -0.12 \\
\hline 31 & $12409.1-730905.8$ & 15.36 & & & 55 & 16.57 & & 15.09 & $13.06^{c}$ & $12.31^{c}$ & 15.37 & -3.73 & 0.01 & -0.28 \\
\hline 32 & $12407.9-730905.1$ & 14.24 & 12.18 & 1.07 & & & & 12.31 & 11.98 & 11.19 & 11.07 & -4.85 & 3.17 & 1.24 \\
\hline 33 & $12408.9-730854.0$ & 16.92 & & & 62 & 18.02 & & 15.34 & 14.88 & 14.64 & 14.53 & -2.17 & 2.39 & 0.71 \\
\hline 34 & $12409.9-730853.7$ & 15.73 & & & 64 & 17.00 & & 16.39 & & 15.76 & 16.23 & -3.36 & -0.30 & 0.16 \\
\hline 35 & $12423.3-730845.7$ & & & & & & & & & & 16.74 & & & \\
\hline 36 & $12422.2-730841.8$ & 15.58 & & & 66 & 16.89 & & & & & 16.33 & -3.51 & -0.75 & \\
\hline 37 & $12421.0-730837.6$ & & & & & & & & & & 16.14 & & & \\
\hline
\end{tabular}

${ }^{p}$ Present work.

$v_{\mathrm{c}}$ Magnitude obtained through an interference filter of $\delta \lambda \sim 100 \AA$ centered at $\lambda 5300 \AA$.

${ }^{m}$ From the Massey survey (2'.32 per pixel). In this survey the crowded stars suffer from a lack of spatial resolution: the red star \#7 is blue and the blue star \#8 is red in our photometry.

${ }^{h}$ Numbering of HM99. $\mathrm{c}^{1}, \mathrm{c}^{2}, \mathrm{c}^{3}, \mathrm{c}^{4}$ and $\mathrm{c}^{5}$ are multiple stars composed of stars named $(7,6),(9,10,11,12,13),(36,38),(71,72)$ and $(46,48)$ by HM99. For these stars, the given magnitude corresponds to the integration of each component.

${ }^{d}$ From the DENIS survey.

${ }^{2 m}$ From the 2 MASS, ${ }^{c}$ probably contamination of \#31 by \#32.

Errors in magnitudes are as follows: $\left(5300 \AA\right.$ A continuum $\left(\right.$ cont $\left._{5300}\right)$.

We find for $\left(v_{\mathrm{c}}\right)^{p}<14.0,14.0<\left(v_{\mathrm{c}}\right)^{p}<16.0$ and $16.0<\left(v_{\mathrm{c}}\right)^{p}<17.5$ a mean standard deviation of $0.01 \mathrm{mag}, 0.03$ mag and 0.06 mag respectively and for $m_{K}<14.5,14.5<m_{K}<15.5$ and $15.5<m_{K}<16.7$ a mean standard deviation of 0.008 mag, 0.016 mag and 0.04 mag respectively, save N88B which is blended in the wing of the bright core N88A (see text).

(Cutri et al. 2003) catalogues. Discrepancies may be seen between the different photometries. The last three columns show the absolute magnitude $M_{V}$ and the $V-K$ and $J-K$ colour index. The magnitude of the faint component \#31 (N88B), although contaminated by the strong wing of the component \#32
(N88A) $\left(F W H M=2^{\prime \prime} .5\right)$ at a distance of $\sim 5^{\prime \prime}$ (Fig. 2 of Paper I), is determined within an error bar of $\sim 0.02 \mathrm{mag}$. In the Massey survey, due to low spatial resolution with a pixel scale of 2 '. 3 , the $H$ and $K$ magnitudes of \#31 are underestimated. N88 (\#32 in Table 2) shows a red $J-H$ colour $(\sim 0.33)$ in contradiction 


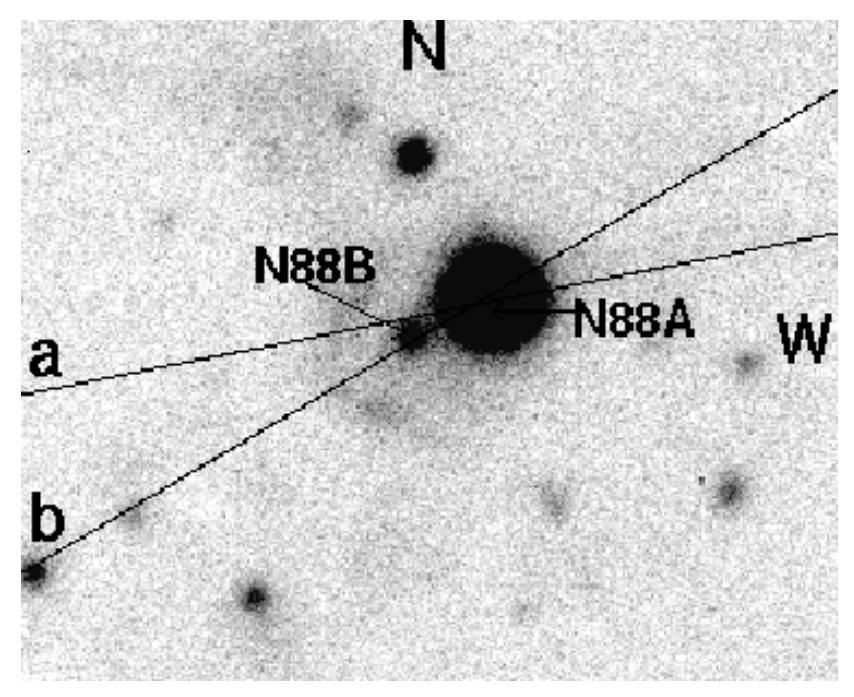

Fig. 2. N88A in the HW 81 field taken through the $2.13 \mu \mathrm{m}$ filter and with sky background subtracted. The black lines labelled $\mathbf{a}$ and $\mathbf{b}$ correspond to the two locations of the slit used in the spectroscopic mode. The field size corresponds to $45^{\prime \prime} \times 39^{\prime \prime}$.

with the blue colour found by Israel \& Koorneef (1991) at low spatial resolution.

\subsection{Spectroscopic observations}

We set out to record spectra of the pure $\mathrm{H}_{2}$ emission shown in the $\mathrm{S}(1) v=1-0$ line in Fig. 1d (Paper I). Spectroscopy of weak $\mathrm{H}_{2}$ emission in the $K$-band is dominated by the problem of removing the sky background. This background consists, at shorter wavelengths up to $\sim 2.2 \mu \mathrm{m}$, of emission from excited $\mathrm{OH}$ in the upper atmosphere, the so-called Meinel bands (Meinel 1950). At longer wavelengths, greater than $\sim 2.4 \mu \mathrm{m}$, thermal emission becomes obtrusive. The Meinel bands and thermal emission can be brighter by respectively two and three to four orders of magnitude than the signals for which we search. Moreover the Meinel bands are variable on a time-scale of minutes. The tactics adopted were to record a spectrum of the object for $100 \mathrm{~s}$ or less, and then nod the slit along its length onto the sky and record again for the same time. The exposure time was chosen partly because of the rapid variability of the Meinel bands and also since a $100 \mathrm{~s}$ exposure gave a signal of less than 20000 counts/s at $2.5 \mu \mathrm{m}$, which lies considerably below the saturation limit of the detector. Spectral photometric calibrations were performed each night with HD 7644 $\left[\alpha(2000)=1^{\mathrm{h}} 15^{\mathrm{m}} 37^{\mathrm{s}} .375, \delta(2000)=-45^{\circ} 26^{\prime} 36.27^{\prime \prime}\right]$ of spectral type F6V (Groenewegen \& Blommaert 1998), which lies in the same region of the sky as the SMC. Spectral images were obtained in the short wavelength range and long slit spectroscopic mode at low and medium resolution (SW-LR and SW-MR) (Table 1). The slit length spans $120^{\prime \prime}$ on the sky, with a pixel scale of 0 .' 146 per pixel. Calibration lamp spectra show that structureless square-topped lines, with a width of 14 pixels, are formed in SW-LR mode with a slit width of $2^{\prime \prime}$ (6 pixels with a slit width of $0 .{ }^{\prime} 6$ ). Due to the small size of the field of interest, a nod throw along the slit of $60^{\prime \prime}$ has been chosen, allowing both object and sky data to be obtained on each spectral
Table 3. The evolved stars in the N88 region.

\begin{tabular}{llllllll}
\hline \hline Id & $J-K$ & $\left(M_{V}\right)_{0}$ & $(V-K)_{0}$ & $T_{\text {eff }}$ & $\log \left(\frac{L}{L_{\odot}}\right)$ & $M_{\odot}$ & $\log (\mathrm{yr})$ \\
\hline 6 & 0.90 & -1.87 & 3.23 & 4100 & 2.68 & 1.2 & 9.8 \\
9 & & -2.26 & 2.91 & 4350 & 2.84 & 2.5 & 8.8 \\
10 & 0.77 & -4.27 & 2.57 & 4750 & 3.65 & 8 & 7.6 \\
12 & 0.64 & & & 4800 & & & \\
13 & 0.44 & -4.50 & 1.40 & 6000 & 3.74 & 8 & 7.6 \\
14 & 1.04 & -2.12 & 3.27 & 4100 & 2.79 & 1.5 & 9.2 \\
16 & 0.68 & -2.89 & 2.27 & 4850 & 3.10 & 5 & 8.0 \\
18 & 0.87 & & & 4100 & & & \\
22 & 0.76 & & & 4350 & & & \\
33 & 0.71 & -2.24 & 2.35 & 4800 & 2.84 & 4 & 8.2 \\
\hline
\end{tabular}

image. Each member of the pair of images corresponding to one nod throw are subtracted from the other. This gives rise to two images, one positive and one negative at different locations on the detector. The subtraction removes the $\mathrm{OH}$ Meinel bands and other telluric features, thermal emission from the sky and any "dark" contribution from the detector. Flat-fielding is obtained in each band using both spectroscopic twilight flats, which provide a correction for the wavelength response of the detection system, and the calibration star, which accounts for the atmospheric transmission. In addition a correction for the blackbody curve corresponding to the known temperature of the calibration star $(6281 \mathrm{~K})$ has been introduced. A single final image is obtained by shifting the images by the $60^{\prime \prime}$ of the nod throw, combining the positive and negative signals. The total exposure time and spectral resolution for spectra recorded with slit orientation $a$ and $b$ (Fig. 2) are shown in Table 1. The fifth to the second orders of the grating were employed to give access to the $Z, J, H$ and $K$ bands in the atmospheric window in which many ro-vibrational $\mathrm{H}_{2}$ emission lines may be found. Incomplete $K$ and $H$ band spectra have also been obtained at medium resolution (Table 1). This has the important advantage, in $K$, that the overlapped He I lines at 2.1120 and $2.1132 \mu \mathrm{m}$ can be resolved from the $\mathrm{S}(1) v=1-0 \mathrm{H}_{2}$ line at $2.121 \mu \mathrm{m}$. This is shown in data presented in Sect. 3.4.2. The 2 " slit was used in position $a$, shown in Fig. 2, chosen to record the spectra in $Z, J$, $H$ and $K$ bands across the bright core of N88A and through the nebulosity to the east, avoiding N88B. The 0 .' $^{\prime} 6$ slit was used in position $b$ to obtain spectra in $K$ and $H$ bands, looking across the bright core N88A and the stellar component N88B.

\section{Results}

\subsection{Evolved stars in the N88 region}

Except for star \#33 (just north of N88, Fig. 1), all the giant stars of $1.44<V-K<3.30$ and $0.64<J-K<0.87$ are located outside the blue cluster HW 81. The temperature $T_{\text {eff }}$ for each star was extracted from the model $T_{\text {eff }}$ versus $V-K$ colour index for GKM giants found in Bessel et al. (1998). They show that the $V-K$ index is the least sensitive to metallicity and, for $T_{\text {eff }}$ greater than $4000 \mathrm{~K}$, is essentially independent of abundance. When $V-K$ was not available (\#12, \#18 and \#22) we used the $J-K$ color. However this colour becomes redder with decreasing metallicity according to Bessel et al. (1998). Table 3 shows 


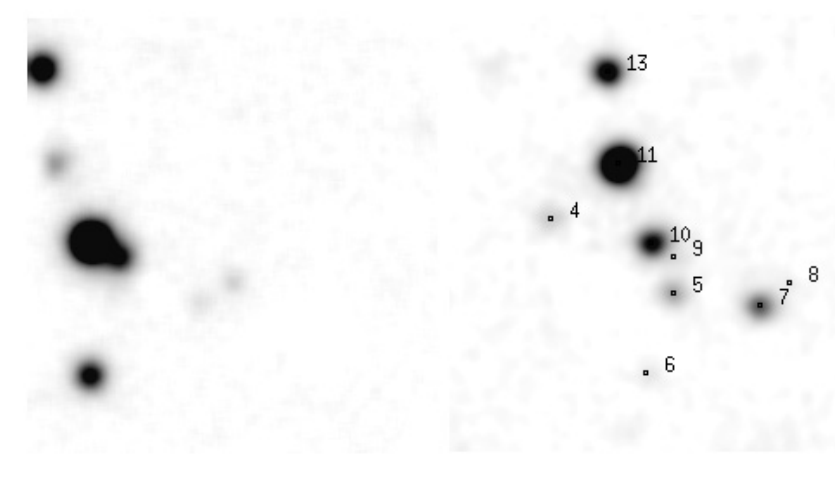

Fig. 3. Images of the cluster HW 82 (Fig. 1) obtained through the $K$ and cont ${ }_{5300}$ filters. HW 82 in the $K$ image (left $)$ is truncated to the east on our original frame. In the cont ${ }_{5300}$ image (right) 9 stars are clearly visible and the numbers refer to Table 2 . For each image the field size is $\left(35^{\prime \prime} \times 40^{\prime \prime}\right)$.

the $T_{\text {eff }}$ as well as $\log \left(L / L_{\odot}\right)$. Three of these stars, \#6, \#9, \#10, lie in the cluster HW 82 (Fig. 3 ) of about $35^{\prime \prime} \times 40^{\prime \prime}$ or $11 \times$ $13 \mathrm{pc}$ (adopting a SMC distance of $\sim 66 \mathrm{kpc}$ (Jacoby et al. 1990)) located $\sim 100^{\prime \prime}$ south-east of N88A (Fig. 1), whereby $1^{\prime \prime}$ on the image corresponds to $0.32 \mathrm{pc}$. In contrast to HW 81 , this cluster is not associated with ionized gas (HM99) and no molecular hydrogen was detected in the infrared, see Paper I. Using a distance modulus $m_{V}-M_{V}=19.09$ (Jacoby et al. 1990), the $M_{V}$ of the evolved stars of the N88 field was found to lie in the range of -1.81 to -4.43 and $\log \left(L / L_{\odot}\right)$ in the range of of 2.68 to 3.74 . From the Geneva evolutionary models (see "http: //webast . ast. obs-mip. fr/stellar") for low-mass, intermediate-mass and massive stars with initial chemical composition $Z=0.004$ (Lejeune \& Schaerer 2001), these stars span the mass range between 1 and $8 M_{\odot}$. Using their isochrones with the same $Z$, we derive a wide range of ages between $40 \mathrm{Myr}$ and $6.0 \mathrm{Gyr}$. The stars were dereddened using the low mean value $[E(B-V)=0.034]$ for the SMC given by Mc Namara \& Feltz (1980). The mass and the age of these evolved stars are listed in Table 3.

\subsection{Stellar content in the cluster HW 82}

Figure 3 shows the cluster HW 82 in the $K$ and cont ${ }_{5300}$ bands where 9 stars are detected. According to Bessel et al. (1998) the stars \#6, \#9, \#10 with a $V-K=2.6-3.6$ should be red giants and \#8, \#13 (with $J-K=0.44$ ) less evolved stars of type $\mathrm{G}$, while stars \#4, \#5, \#7 and \#11 from $M_{V}$ are classified as blue stars. According to Vacca et al. (1996), based upon a derived integrated $M_{V}$ of $\sim-5.38$, star \#11 should be equivalent to an $\mathrm{O} 5 \mathrm{~V}$ star or five $\mathrm{O} 8 \mathrm{~V}$ stars. The single stars \#4, \#5 and \#7 $\left(-3.7<M_{V}<-2.3\right)$ should be classified as early B stars (Schmidt-Kaler 1982). This is confirmed by the HM99 colour-magnitude diagram where stars \#4, \#5, $\# 7$ as well as the five components of \#11 are located at the extreme left. HW 82 appears composed of a group of main sequence massive stars, several evolved stars of intermediate age and old stars (40 Myr-6 Gyr). The low mass supergiant

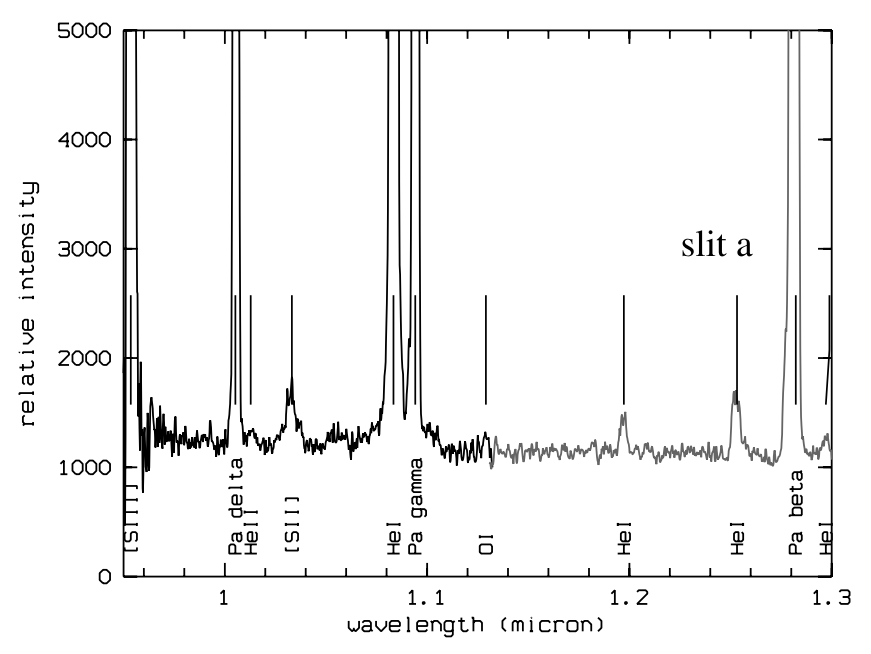

Fig. 4. $Z J$ spectrum of N88A in the range $0.95 \mu \mathrm{m}-1.3 \mu \mathrm{m}$ (slit a) produced by merging $Z$ and $J$ spectra for this object. The spectral resolution $\delta \lambda / \lambda$ is $\sim 350$.

stars \#10 and \#13 could have dispersed the gas (Genzel \& Stutzki 1989) and could explain the absence of ionized gas in the region (HM99). The stellar content of the N88 region suggests that it is composed of several different star formation events which are not physically associated. In this respect, N88 is comparable to N81, a similar neighbouring region (HM99).

\subsection{Extinction in $N 88 A$}

We revisit here the computation of the visual extinction using the new $2^{\prime \prime}$ slit $Z$ and $J$ spectra. $Z$ and $J$ described in the present paper are properly matched, using the $\mathrm{P} \gamma$ line common to the two overlapping spectra, giving a single spectrum (ZJ) in the range of $0.95-1.3 \mu \mathrm{m}$. The resulting spectrum is seen in Fig. 4. We use the average extinction law of Cardelli et al. (1989). This law is assumed to be valid for optical and nearIR regions even in galaxies of different metallicity (Petersen \& Gammelgaard 1996). Column 4 in Table 4 shows the flux of the hydrogen lines $\mathrm{P} \delta$, and $\mathrm{P} \beta$ relative to $F(\mathrm{P} \gamma)=1$ extracted from the $Z J$ spectrum in Fig. 4. The reddening in $Z J$ is derived from the slope of a linear fit of the data points in the form $2.5 \log \left(R_{\mathrm{o}} / R_{\mathrm{p}}\right)$ as a function of $A_{\lambda} / A_{\mathrm{V}}$ for $R_{V}=3.1$ (Petersen $\&$ Gammelgaard 1996). We find a mean $A_{\mathrm{V}}=1.52 \pm 0.1$. This value is slightly greater than 1.38 and 1.40 determined in Paper I and by Kurt et al. (1999) through a 2 " slit and a 3700-7400 wavelength range. The derivation of the extinction in an object of such complex and dusty morphology as N88A (HM99) is certainly very dependent on the slit width, and higher spatial and spectral resolution are required for a better determination.

\subsection{Spectroscopy along slits $a$ and $b$}

Data extracted from the spectral images at slit positions $a$ and $b$ (Fig. 2) for chosen wavelengths, corresponding to $\mathrm{H}_{2} 1-0 \mathrm{~S}(1)$, $\mathrm{HeI}(2.06 \mu \mathrm{m})$ and $\mathrm{Br} \gamma$, are shown in Figs. 5a and 6 respectively. There is a great variety of structures, including several abrupt rims to the east and smoother behaviour to the west of N88A. The former correspond to ionization fronts and/or 

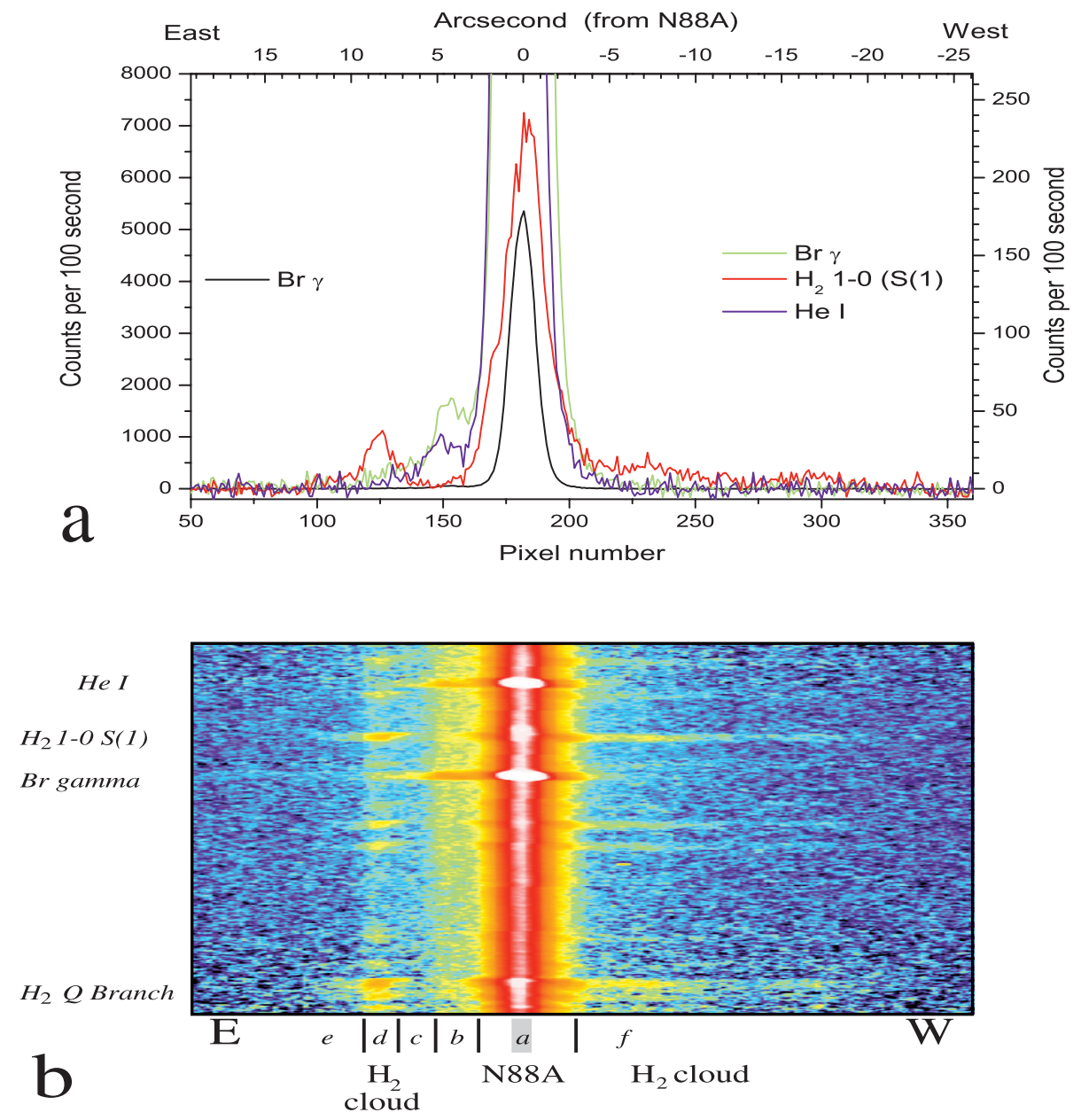

Fig. 5. Upper frame: intensity distribution along the slit $a$ (low resolution): $\mathrm{Br} \gamma$ line (green line), $\mathrm{He} \mathrm{I}$ at $2.06 \mu \mathrm{m}$ (blue line) and pure $\mathrm{H}_{2}$ $v=1-0 \mathrm{~S}(1)$ emission (red line) [all obtained from 11 pixel integration with subtraction of a nearby continuum for the same amount]. The lefthand scale is fixed to the $\mathrm{Br} \gamma$ line (black line), the righthand one is expanded to show fainter details in the 3 lines. East is to the left. Lower frame: identification of regions (see text). The contrast is enhanced using a $3 \times 3$ boxcar smoothing.

Table 4. Normalized observed line fluxes for Paschen lines extracted from the $Z$ and $J$ spectra.

\begin{tabular}{cllll}
\hline \hline $\mathrm{Id}$ & $\begin{array}{l}\lambda \\
(\AA)\end{array}$ & $\frac{A_{\lambda}}{A_{\mathrm{V}}}$ & $\begin{array}{l}\left(\frac{F \lambda}{F(\operatorname{Pa\gamma })}\right)_{\mathrm{o}} \\
(\text { slit 2 })^{\prime \prime}\end{array}$ & $\left(\frac{I \lambda}{I(\operatorname{Pa\gamma }))_{\mathrm{p}}}\right.$ \\
\hline $\mathrm{P} \delta$ & 10049 & 0.401 & 0.575 & 0.61 \\
$\mathrm{P} \gamma$ & 10938 & 0.350 & 1. & 1 \\
$\mathrm{P} \beta$ & 12818 & 0.271 & 2.02 & 1.79 \\
\hline$A_{\mathrm{V}}$ & & & 1.52 & \\
\hline
\end{tabular}

Column 3: $\frac{A_{\lambda}}{A_{\mathrm{V}}}$ derived from Eqs. (2) and (3) of Cardelli et al. (1989); Column 4: ${ }_{\mathrm{o}}$ observed emission line flux ratios through a $2^{\prime \prime}$ long slit; Column 5: p predicted ratios derived from the case B of Hummer \& Storey (1987) for $N_{\mathrm{e}}=100 \mathrm{~cm}^{-3}$ and $T_{\mathrm{e}}=10^{4} \mathrm{~K}$.

the action of outflows from N88A. This is discussed below for each slit. In order to obtain the pure emission for each line considered in Figs. 5a and 6, a continuum background signal at a position parallel to the slit and at the nearest available wavelength free from any other line emission has been subtracted.

\subsubsection{Features found along slit $a$, outside the core region}

Figure $5 \mathrm{~b}$ shows the spectral image from which data in Fig. 5a were obtained. There are 5 distinctive regions, a-e, to the east of N88A, shown at the base of Fig. 5b. These regions are as follows:

(a) the central core of N88A, showing a bright continuum with $\mathrm{HeI}, \mathrm{HI}$ and $\mathrm{H}_{2}$ lines superimposed;

(b) a region showing a faint continuum with $\mathrm{HI}$ and $\mathrm{HeI}$ lines. In this region a pedestal of $\mathrm{HI}$ emission extends from $3^{\prime \prime}$ to $6^{\prime \prime}$ ( 1 to $2 \mathrm{pc}$ ) from the central bright core of N88A. The $\mathrm{HI}$ and HeI lines lie over a faint continuum clearly visible in Fig. 5 b but subtracted in Fig. 5a. At the eastern end of this region (at about $55^{\prime \prime} 3$ or $1.7 \mathrm{pc}$ ) $\mathrm{Br}_{\gamma}$ emission is reduced to $1 \%$ of its peak value and then smoothly declines extending as far as $10^{\prime \prime}(3.1 \mathrm{pc})$. For the $\mathrm{H}_{2}$ emission, the relative decline in signal is at first less rapid (between regions a and $\mathrm{b}$ ) than $\mathrm{Br} \gamma$ emission, but $\mathrm{H}_{2}$ falls rapidly to zero thereafter in region $b$;

(c) a region without continuum but with weakly declining $\mathrm{HI}$ and HeI lines; 


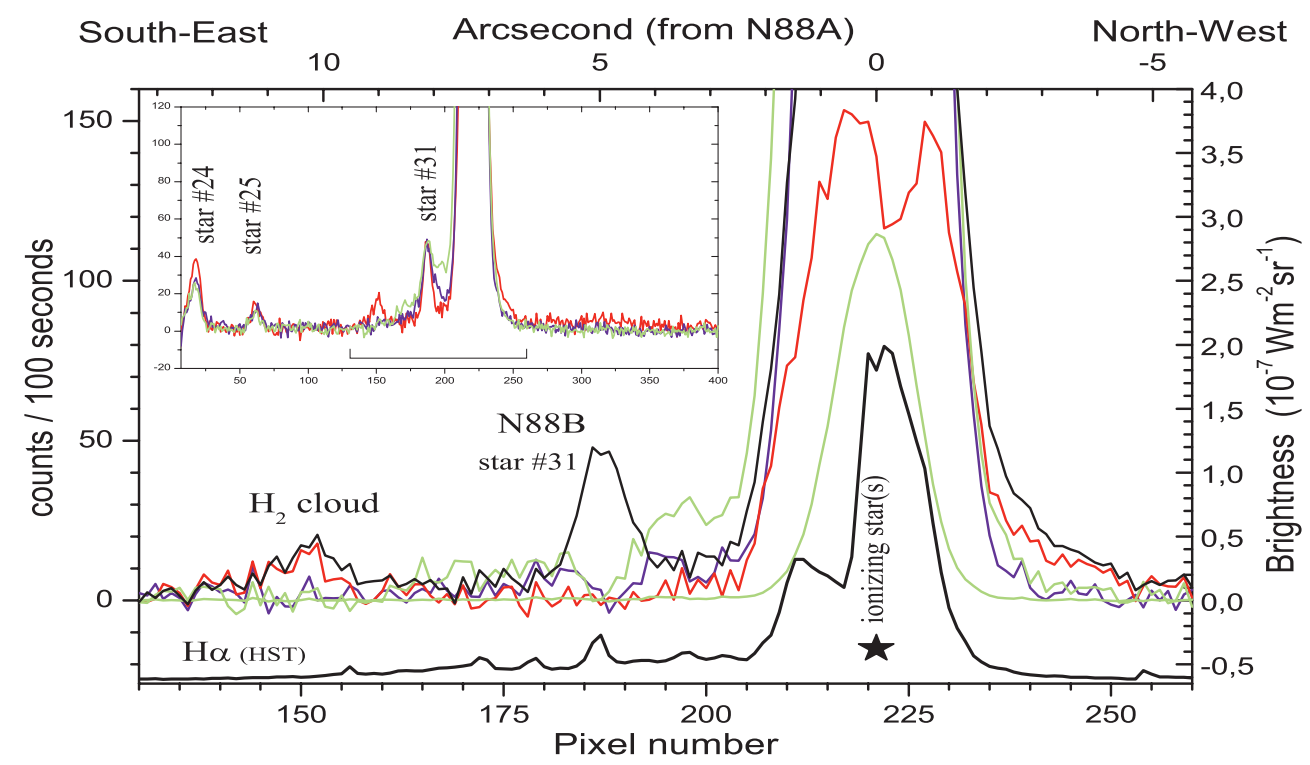

Fig. 6. Intensity distribution along the slit $b$ (medium resolution), crossing N88B and N88A: Br $\gamma$ line (light lines: the lower one is the total signal - not to scale), He I at $2.06 \mu \mathrm{m}$ (dark grey line) and pure $\mathrm{H}_{2} v=1-0 \mathrm{~S}(1)$ emission (grey line). $\mathrm{H}_{2}+$ continuum at $2.13 \mu \mathrm{m}$ (black line). All obtained from 7 pixel integration with subtraction of a nearby continuum also integrated over 7 pixels. The inset shows the same data without subtraction of this nearby continuum (same colour coding). A cross section corresponding to the same orientation and same slit width, extracted from HST H $\alpha$ archives data is overplotted (black line at the bottom, in arbitrary units and with negative offset). The south-east is to the left and the horizontal scale spans $19^{\prime \prime}$. The horizontal scale spans $58^{\prime \prime}$ for the inset.

(d) a region with $\mathrm{H}_{2}$ emission and still declining $\mathrm{HeI}$ or $\mathrm{H}$-atom lines. The most remarkable characteristics of this last region are the abrupt edges, particularly to the east, which are clearly visible in Fig. 5b. Region d delimits an $\mathrm{H}_{2}$ cloud lying at a distance of about 6.' 4 to 9.'3 (2.0 to $3.0 \mathrm{pc}$ ) from N88A. This cloud may be seen in Fig. $5 \mathrm{~b}$, in numerous $\mathrm{H}_{2}$ emission lines (where only the 1-0 S(1) line and the Q-branch are identified in Fig. 5b, for clarity);

(e) This region marks the edge of the $\mathrm{H}_{2}$ cloud. To the east, the $\mathrm{HI}$ and $\mathrm{Br} \gamma$ emissions cease entirely while the $\mathrm{H}_{2}$ emission remains as a faint declining signal extending up to 12 .'5 $(4.0 \mathrm{pc})$. To the west of N88, the morphology displays only 2 regions: $(a)$ the central core and $(f)$ an extended $\mathrm{H}_{2}$ emission region, without any $\mathrm{HeI}$ or $\mathrm{H}$-atom emission outside the central core. The $\mathrm{Br} \gamma$ emission decreases to $1 \%$ of its peak value at a distance of $3^{\prime \prime}(1.0 \mathrm{pc})$ and ceases almost completely at $\sim 5^{\prime \prime} .5$ from the core of N88A. $\mathrm{H}_{2}$ emission declines to $\sim 6 \%$ at a distance of $3.6^{\prime \prime}$. Thereafter there is very weak emission, clearly visible in Fig. $5 \mathrm{~b}$ in various $\mathrm{H}_{2}$ emission lines. Emission may be detected in the $\mathrm{S}$ (1) 1-0 line, as far as $22^{\prime \prime}$ (7 pc).

Despite the low spatial resolution, arising from the PSF of 1'.3 for data in Fig. 5, we are able to determine that the $\mathrm{H}_{2}$ emission shows a greater width in the core than the $\mathrm{Br} \gamma$ emission, suggesting that the core may contain several objects. Higher resolution data, reported in the next section, reveal this structure.

\subsubsection{Features along slit $b$, at medium resolution}

Just as for slit $a$, slit $b$ is centered on the N88A core but differs in crossing N88B and the stars \#24 and \#25 to the south-east (Figs. 1 and 2). The spatial distribution of emission along 58"

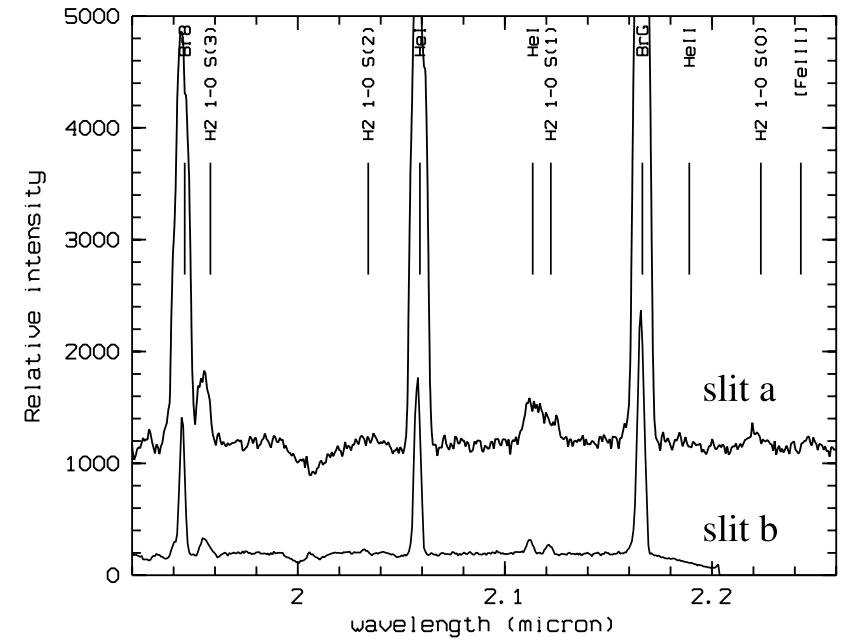

Fig. 7. $K$-band spectrum of N88A from $1.9 \mu \mathrm{m}$ to $2.2 \mu \mathrm{m}$. The thick line, marked slit a, shows a spectrum obtained with a spectral resolution $\delta \lambda / \lambda \sim 320$. A spectrum at higher resolution $\delta \lambda / \lambda \sim 650$, marked slit $\mathrm{b}$, is shown as a thin line and resolves the two HeI lines $\lambda 2.1120$, 2.1132 and the $\mathrm{H}_{2} \mathrm{~S}(1)$ line at $\lambda 2.1213$.

of the slit, shown in the inset of Fig. 6 bears some similarity to that within slit $a$.

A noticeable difference is that along the direction of slit $b$, the $\mathrm{Br}_{\gamma}$ pedestal and other $\mathrm{H}$-atom lines, as well as the faint underlying continuum, are not detected between N88A and N88B. The higher spectral resolution data associated with slit $b$ allow the resolution of the $\mathrm{H}_{2}$ line at $2.121 \mu \mathrm{m}$ from the $\mathrm{He} \mathrm{I}$ line at $2.113 \mu \mathrm{m}$. Both $\mathrm{H}_{2}$ and $\mathrm{He}$ emit in the core, as illustrated in the lower spectrum in Fig. 7. In addition to the spectral resolution provided by a 0 . $^{\prime} 6$ slit, an improved seeing of $0 .{ }^{\prime} 8$ 
during these observations led to a better spatial resolution. This in turn allowed the observation of structure inside the core in $\mathrm{H}_{2}$ (red line in Fig. 6), which is absent in $\mathrm{Br}_{\gamma}$ (lower green line). It appears therefore that the core is composed of two distinct regions of $\mathrm{H}_{2}$ emission. A hint of this structure already appeared in the low resolution spectra (Fig. 5a), observed in slit $a$. A gap of about 1 .'5 (0.4 pc) separates the two $\mathrm{H}_{2}$ IR emission maxima in Fig. 6. There is no such counterpart in HI emission which shows a peak located on the brightest $\mathrm{H}_{2}$ emission peak. The observed gap very likely corresponds to the dark lane previously observed in imaging by Kurt et al. (1999) and HM99 with the HST. Using H $\alpha$ data of HM99 retrieved from http://archive.stsci.edu, we have computed the intensity distribution at the same slit location and orientation as that associated with slit $b$ (Fig. 6). The peak $\mathrm{H} \alpha$ emission in the HST data, however, corresponds in position to our present $\mathrm{Br} \gamma$ peak where we find the actual physical gap in $\mathrm{H}_{2}$ emission. This suggests that the conditions which excite $\mathrm{H}$ atoms most effectively are distinct from those which exite $\mathrm{H}_{2}$, in keeping with a photodissociation region model as a primary mechanism for excitation of $\mathrm{H}_{2}$ in this region (see Sect. 3.6). We also note that there are uncertainties which may amount to $\sim 0.5$ ( 3 pixels) in the slit position relative to the object, which may contribute to the relative shift of $\mathrm{H}_{2}$ and $\mathrm{H}$ emission. This could be improved, given the less than 0.' 4 angular size of the very bright inner part of the N88A core (HM99), by using the higher spatial resolution of the NACO adaptive optics instrument on UT4 (86 mas slit). The pure $\mathrm{H}_{2}$ emitting cloud is detected in slit $b$ to the south-east of the N88A core but is less bright than in slit a and lies in the present case at a distance of about 9.' 3 to 11 ". 1 (2.5 to $3 \mathrm{pc}$ ) from N88A. Moving along slit $b$, to the north-west, weak $\mathrm{H}_{2}$ emission extends to about $25^{\prime \prime}(6.8 \mathrm{pc})$, much as observed in slit $a$. The spectrum of N88B obtained using slit $b$ shows only a pure continuum at our detection limit. Thus N88B appears to be a star characterized through this continuum emission.

\subsection{K-band spectra in regions $a, b$ and d (Fig. 5b)}

The complete spectrum spanning the $Z, J H$ and $K$ bands from 0.98 to $2.5 \mu \mathrm{m}$ (Figs. 4,7 and 8 ) is dominated by $\mathrm{H}$ and $\mathrm{He}$ atomic lines lying above the strong continuum of the N88A core. Faint $\mathrm{H}_{2}$ molecular lines were detected superimposed on the strong continuum in the $K$ band only. Turning to the $\mathrm{H}_{2}$ emission in more detail, spectra obtained at three different locations are shown in Fig. 9. For each spectral line, the nearest position on the slit free of any $\mathrm{H}_{2}$ emission has been subtracted in order to reject the very weak background covering the whole N88 region. The upper spectrum, labelled 1 in Fig. 9, shows the $K$ band spectrum in the N88A core (region a in Fig. 5b). Both atomic lines and molecular $\mathrm{H}_{2}$ lines are present, overlying a bright continuum. The lower spectrum, labelled 3, is obtained in the brightest part of the eastern cloud (region $\mathrm{d}$ in Fig. 5b) about 8 pc from N88A. This spectrum shows pure $\mathrm{H}_{2}$ emission only, except for the $\mathrm{Br}_{\gamma}$ line. $\mathrm{H}_{2}$ line brightnesses for spectrum 3 are reported below in Table 5. Only $\mathrm{S}(1)$ and $\mathrm{S}(0) v=1-0$ and $\mathrm{S}(1) v=2-1$ are free of atmospheric absorption. Spectrum 2 in Fig. 9 was obtained in the

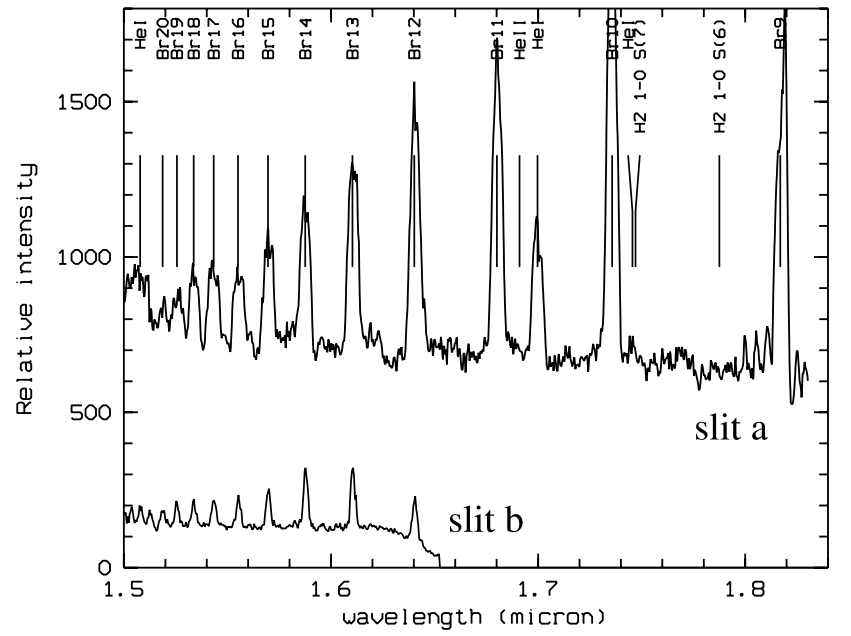

Fig. 8. $H$-band spectrum of N88A from $1.5 \mu \mathrm{m}-1.8 \mu \mathrm{m}$ (slit a). The upper spectrum has a resolution $\delta \lambda / \lambda \sim 300$ and the lower (slit b) a resolution $\delta \lambda / \lambda \sim 650$.

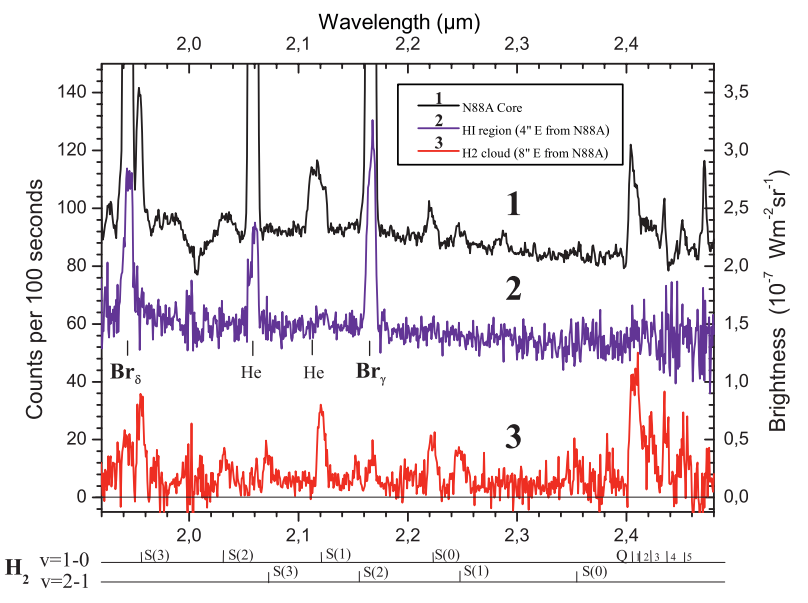

Fig. 9. Spectral images in the $K$-band in slit a, at three different locations (from top to bottom of the figure): 1) On the N88A core (intensities shifted by +250 counts, then divided by 10). 2) North of N88B (4" from N88A, intensities shifted by +40 counts). 3) $\mathrm{H}_{2}$ nebulosity (8" from N88A, scale as shown): see Table 5 for line brightness values.

intermediate region (region b in Fig. 5b) about 4 pc from N88A. The main characteristic of spectrum 2 is the absence of any trace of molecular $\mathrm{H}_{2}$ emission, showing only $\mathrm{H}$ and $\mathrm{He}$ lines lying on a faint continuum.

\subsection{Excitation mechanisms of $\mathrm{H}_{2}$}

Our present data are too noisy (see Fig. 9) and contain insufficient lines unaffected by atmospheric absorption to extract a reliable effective temperature for the $\mathrm{H}_{2}$ cloud. Notwithstanding, we may make a qualitative comparison of relative intensities of $\mathrm{H}_{2}$ emission lines for spectra taken at different positions. Thus, if we compare the relative intensities in Table 5, from spectrum 3 in Fig. 9 with those obtained from spectrum 1 in the same figure, then within the $S / N$ of our data these ratios are the same. Since line ratios are diagnostic of physical conditions (see below), we conclude that the $\mathrm{H}_{2}$ emission from the core region, spectrum 1 , shares a common excitation 
Table 5. $\mathrm{H}_{2}$ emission line brightness, extracted from spectrum 3 of Fig. 9, in units of $10^{-9}$ Watt $\mathrm{m}^{-2} \mathrm{sr}^{-1}$. Note that $\mathrm{Q}$ branch lines and S(3) 1-0 in particular may be affected by atmospheric absorption: see Sect. 3.6. Ratios of brightness relative to $\mathrm{S}(1) v=1-0$ are shown in the right-hand column.

\begin{tabular}{ccrr}
\hline \hline $\mathrm{H}_{2}$ line & $\lambda(\mu \mathrm{m})$ & Brightness & Ratio \\
\hline $\mathrm{S}(3) 1-0$ & 1.9570 & 67.5 & 1.06 \\
$\mathrm{~S}(4) 2-1$ & 2.0035 & & \\
$\mathrm{~S}(2) 1-0$ & 2.0332 & 22.5 & 0.35 \\
$\mathrm{~S}(3) 2-1$ & 2.0729 & 17.5 & 0.27 \\
$\mathrm{~S}(1) 1-0$ & 2.1213 & 63.8 & 1 \\
$\mathrm{~S}(2) 2-1$ & 2.1536 & 14 & 0.22 \\
$\mathrm{~S}(0) 1-0$ & 2.2227 & 37.5 & 0.59 \\
$\mathrm{~S}(1) 2-1$ & 2.2471 & 23 & 0.36 \\
$\mathrm{~S}(0) 2-1$ & 2.3550 & & \\
$\mathrm{~S}(1) 3-2$ & 2.3858 & & \\
$\mathrm{Q}(1) 1-0$ & 2.4059 & 94 & 1.47 \\
$\mathrm{Q}(2) 1-0$ & 2.4128 & & \\
$\mathrm{Q}(3) 1-0$ & 2.4231 & 35 & 0.55 \\
$\mathrm{Q}(4) 1-0$ & 2.4368 & 45 & 0.71 \\
\hline
\end{tabular}

mechanism with the eastern cloud, $8 \mathrm{pc}$ distant from the core, whose spectrum is that of spectrum 3 in Fig. 9. The two spectra (not shown) obtained in the eastern cloud in both slit $a$ at $4^{\prime \prime}(1.3 \mathrm{pc})$ from N88A (see also Fig. 5) and in slit $b$ at $6^{\prime \prime}$ $(\sim 2.0 \mathrm{pc})$ also exhibit the same line ratios within observational error. Thus we are very probably tracing the same cloud at two different positions in the two slits, about 4 " (1.3 pc) apart. Similarly, despite a poorer $S / N$ ratio, the very faint spectrum emitted in the western nebulosity also looks very similar to the spectrum of the eastern cloud (spectrum 3, Fig. 9), again suggesting a common excitation mechanism. The $v=$ $1-0 \mathrm{~S}(1) / v=2-1 \mathrm{~S}(1)$ line ratio, $R$, is a useful diagnostic of $\mathrm{H}_{2}$ excitation mechanisms in lower density $\left(10^{3}\right.$ to $\left.<10^{5} \mathrm{~cm}^{-3}\right)$ conditions (Sternberg \& Dalgarno 1989). As mentioned above, these $S(1)$ lines are almost free of atmospheric absorption. Moreover, differential reddening should make an unimportant contribution (Kristensen et al. 2003). The line ratio of 0.36 shown in Table 5, equivalent to $R \sim 2.8$, would suggest that a major part of the emission emanates from a low density PDR (Black \& van Dishoeck 1987), for which a value of 2 may be appropriate. This is reminiscent of Orion, in which the $\mathrm{H}_{2}$ emission is dominated by PDRs, but with a significant contribution from very small, highly concentrated and very bright shocked zones e.g. in OMC1 in which the value of $R$ is typically $\sim 7$ (see Gustafsson et al. 2003; Vannier et al. 2001, and references therein). In addition, the line brightness of $\sim 6 \times$ $10^{-8} \mathrm{Wm}^{-2} \mathrm{sr}^{-1}$ in $v=1-0 \mathrm{~S}(1)$ (Table 5) is typical of a weak PDR (e.g. Field et al. 1998). The ratio of the $S(1)$ and $\mathrm{S}(0) v=1-0$ line brightness, $R_{\mathrm{o} / \mathrm{p}}$, supplies further evidence in favour of a low density PDR as a major source of the $\mathrm{H}_{2}$ emission. Data in Table 5 show that $R_{\mathrm{o} / \mathrm{p}}$ has the low value of $\sim 1.7$. Results in Sterberg \& Dalgarno (1989) show that values of $R_{\mathrm{o} / \mathrm{p}}$ in this range may be found in low density PDRs $\left(\sim 10^{3} \mathrm{~cm}^{-3}\right)$. Considerably higher values are typical of C- or J-type shocks,

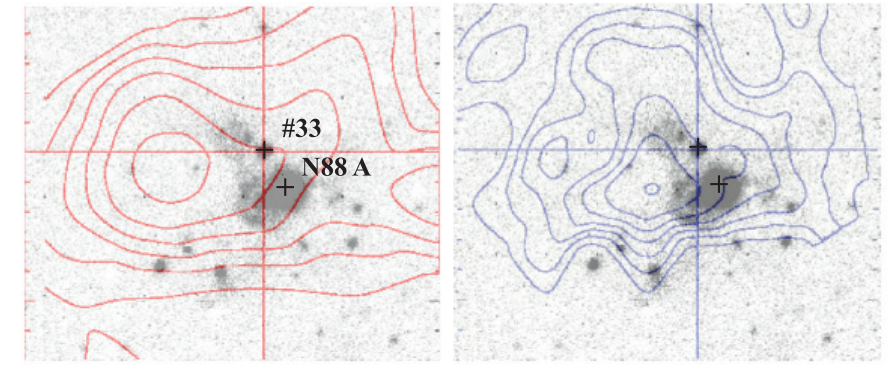

Fig. 10. ${ }^{12} \mathrm{CO}$ contours (Israel et al. 2003) superimposed on our image at $2.12 \mu \mathrm{m}$. Coordinates of these $\mathrm{CO}$ maps are centered on the star \#33, NE from N88A (see Fig. 1). Emission for CO $J=2-1$ [230 GHz] (righthand figure) and CO $J=1-0$ [115 GHz] (lefthand figure). Image scale: $84^{\prime \prime} \times 72^{\prime \prime}$.

in which the ratio may typically approach 6 , the high temperature limit corresponding to an ortho:para ratio of 3 (Wilgenbus et al. 2000).

We note that $\mathrm{H}_{2}$ lines were not detected in the $H$ band, where emission originates from high vibrational states, $v=$ 5-10. Emission from these levels would in principle provide evidence of a photodissociation region (PDR) mechanism for $\mathrm{H}_{2}$ excitation. However in practice no line in the $H$-band is likely to exceed about $30 \%$ of the already rather weak S(1) 2-1 emission, judging from emission spectra recorded in the well-known region NGC 2023 (Burton et al. 1988). Thus detection of $\mathrm{H}_{2}$ in the $H$-band, which is an important diagnostic of the presence of a PDR, must await future observations employing considerably longer integration times.

\subsection{Comparison with $\mathrm{CO}$ observations}

The $\mathrm{H}_{2}$ emission borders the peak flux of an extended CO cloud detected by Israel et al. (2003). Figure 10 shows their ${ }^{12} \mathrm{CO}$ contours for $J=1-0$ and $J=2-1$ superimposed on our image at $2.12 \mu \mathrm{m}$. The significant point is that the peak of the $\mathrm{CO}(2-1)$, tracing warmer gas, lies closer to the possible exciting source, N88A (to the west), than the peak of the CO (1-0), tracing cooler gas. The pure excited $\mathrm{H}_{2}$ lies closer still. Thus the closer one moves towards the core, the greater the excitation. The morphology of $\mathrm{H}_{2}$ and $\mathrm{CO}$ emission therefore provides further support for PDR fluorescent excitation of $\mathrm{H}_{2}$ in this region located $\sim 8^{\prime \prime}(2.6 \mathrm{pc})$ from N88A.

\section{Conclusions}

We summarize our results as follows:

- $V-K$ photometry extended to the whole N88 region reveals a rich population of red giants spanning a range of ages (40 Myr to 6 Gyr) and masses $\left(1.2<M_{\odot}<8\right)$, mainly concentrated in the cluster HW 82 . The presence in this cluster of the supergiant stars \#10 and \#13 could be a possible explanation of the absence of gas in this region. We found for the component \#11 an integrated spectral type equivalent to an $\mathrm{O} 5 \mathrm{~V}$ star. 
- In N88A from the range of wavelengths involving $\mathrm{P} \delta$-P $\beta$, $A_{\mathrm{V}}$ appears $\sim 0.15$ mag greater than $A_{\mathrm{V}}$ previously derived from $\mathrm{H} \alpha / \mathrm{H} \beta$ ratio.

- Spectroscopic observations have confirmed the presence of an $\mathrm{H}_{2}$ molecular cloud, seen in $K$-band ro-vibrational emission, of about $25^{\prime \prime} \times 40^{\prime \prime}$ centered on the young cluster HW 81 and at the western edge of the intensity peak of the small CO cloud recorded in Israel et al. (2003).

- Within observational errors, the relative intensities of the $\mathrm{H}_{2}$ emission lines are very similar at different slit locations, indicating a common excitation mechanism. The origin of the $\mathrm{H}_{2}$ emission is dominated by the UV photon excitation mechanism and the existing evidence points to the $\mathrm{H}_{2}$ emission deriving from regions containing low density gas $\left(10^{3} \mathrm{~cm}^{-3}\right.$ or less).

- $\mathrm{H}_{2}$ infrared emission inside the N88A core shows a physical gap located $\sim 1^{\prime \prime}$ away from the gap observed in $\mathrm{H} \alpha$ with the HST (HM99). Higher resolution imaging and spectroscopy would shed light on this interesting morphology and demonstrate the relationship between the $\mathrm{H}$ and $\mathrm{H}_{2}$ emitting zones and their respective structures.

In conclusion, our data reveal new features of this metal-poor star-forming region. Instrumentation with both high spatial and spectral resolution, such as NACO on UT4 at the VLT, is required to widen our understanding of this archetypal zone in the SMC.

Acknowledgements. J.L.L. and G.T. would like to acknowledge support of the national PCMI program, funded by the CNRS. D.F. would like to acknowledge the support of the Aarhus Centre for Atomic Physics (ACAP), funded by the Danish Basic Research Foundation. We would also like to thank the Director and Staff of ESO for making possible the observations reported in this paper.

\section{References}

Bessel, M. S., Castelli, F., \& Plez, B. 1998, A\&A, 333, 231

Black, J. H., \& van Dishoeck, E. F. 1987, ApJ, 322, 412

Burton, M. J., Howe, J. E., Geballe, T. R., \& Brand, P. W. J. L. 1988, PASA, 15, 194
Cardelli, J. A., Clayton, G. C., \& Mathis, J. S. 1989, ApJ, 345, 245

Cioni, M.-R., Loup, C., Habing, H. J., et al. 2000, A\&AS, 144, 235

Cutri 2003, University of Massachusetts and Infrared Processing and Analysis Center (IPAC) California institute of technology

Davies, R. D., Elliott, K. H., \& Meaburn, J. 1976, MNRAS, 81, 89

Field, D., Lemaire, J. L., Pineau des Forêts, G., Gerin, M., et al. 1998, A\&A, 333, 280

Genzel, R., \& Stutzki, J. 1989, ARA\&A, 27, 41

Groenewegen, M. A. T., \& Blommaert, J. A. D. L. 1998, A\&A, 332, 25

Gustafsson, M., Kristensen, L. E., Clenet, Y., et al. 2003, A\&A, 411, 437

Henize, K. G. 1956, ApJS, 2, 315

Heydari-Malayeri, M., Lebertre, T., \& Magain, P. 1988, A\&A, 195, 230

Heydari-Malayeri, M., Charmandaris, V., Deharveng, L., Rosa, M. R., \& Zinnecker, H. 1999, A\&A, 347, 841

Heydari-Malayeri, M., \& Testor, G. 1982, A\&A, 111, L11

Hodge, P. W., \& Wright, F. W. 1974, AJ, 79, 858

Hummer, D. G., \& Storey, P. J. 1987, MNRAS, 224, 801

Israel, F. P., \& Koorneef, J. 1988, A\&A, 190, 21

Israel, F. P., \& Koorneef, J. 1991, A\&A, 248, 404

Israel, F. P., Johansson, L., Rubio, M., et al. 2003, A\&A, 406, 817

Jacoby, G. H., Ciardullo, R., \& Walker, A. R. 1990, ApJ, 365, 471

Kristensen, L. E., Gustfsson, M., Field, D., et al. 2003, A\&A, 412, 727

Kurt, C. M., Dufour, R. J., Garnett, D. R., et al. 1999, ApJ, 518, 246

Lejeune, T., \& Shaerer, D. 2001, A\&A, 366, 538

McNamara, D. H., \& Feltz, K. A. 1980, PASP, 92, 587

Massey, P. 2002, ApJS, 141, 81

Meinel, A. B. 1950, ApJ, 111, 555

Petersen, L., \& Gammelgaard, P. 1996, A\&A, 308, 49

Schmidt-Kaler, T. 1982, Landoldt-Bornstein, New Series, Group VI, 14

Stanghellini, L., Villaver, E., Shaw, R., \& Mutchler, M. 2003, ApJ, 598,1000

Sternberg, A., \& Dalgarno, A. 1989, ApJ, 338, 197

Testor, G. 2001, A\&A, 372, 667

Testor, G., \& Pakull, M. 1985, A\&A, 145, 170

Testor, G., Lemaire, J. L., \& Field, D. 2003, A\&A, 407, 905

Vacca, W. D., Garmany, C. D., \& Shull, J. M. 1996, ApJ, 460, 914

Vannier, L., Lemaire, J. L., Field, D., et al. 2001, A\&A, 366, 651

Wilcots, E. M. 1994b, AJ, 108, 1674

Wilgenbus, D., Cabrit, S., Pineau des Forêts, G., \& Flower, D. R. 2000, A\&A, 356, 1010 